\title{
Towards an Intelligent Distributed Conveyor
}

\author{
Ioannis Georgilas*, Andrew Adamatzky, and Chris Melhuish \\ Bristol Robotics Laboratory, T Block, BS16 1QY, Bristol, United Kingdom \\ giannis.georgilas@brl.ac.uk http://www.brl.ac.uk/
}

Manipulation, orientation and handling of components, subparts and finished products is an essential element of any industrial process, which attracts substantial portion of resources and infrastructure. A classic manipulation, e.g. robot arms, is not always efficient at the micro-scale and suffers in terms of scaleability. We proposed an alternative concept of manipulation based on an array of actuators. The proposed system rely on the natural concepts of scaleability and parallelism: ciliary motion and excitable media control.

Cilia can be found in every simple micro-organism, while similar structures also exist in bigger ones, like anemones or Infusoria. Either as fixed or pseudolimbs, those structures allow a great degree of simultaneous manipulation, configurability and robustness. As units they are simple and can not apply great forces, while when combined, complex motions and complicated tasks can be achieved. Based on this operational observation we envisage that using an array of simple actuators [1] complex configurability and distributed operation can be achieved; most common actuator solutions are air-jets [2] and microfabricated polyamide [3]. In this work we propose a parallel actuator array, where the actuators are flat, round, vibrating motors, Fig. 1(a)(i), and by the novel mechanical design, using a silicon membrane, Fig. 1(a)(ii), cilia-like manipulation forces are generated. By implementing a modular, distributed hardware design, the cost for each cell remains low and the complexity of the system is not affected by the increase of active surface size. The modules can be connected together in big stacks, Fig. 1(b)(i), in a simple PnP way, since all the electronics are embedded in a compact design, Fig. 1(a)(iii).

Excitable media are multi-state systems that when excited externally, a wavelike propagation of state alterations appear. It has been proven that excitable media can be used for computation purposes [4] proving to be universal processing systems, with parallel computing abilities since the entire surface interacts simultaneously. Typical excitable media used for robot control are reaction-diffusion chemicals [5] and Cellular Automata (CA) [6]. We imitate excitable media using CA which each cell takes there states: resting $(\cdot)$, refractory $(-)$ and excited $(+)$. The CA is coupled with the hardware prototype. We use a particular excitable CA called $2^{+}$-medium [4] to map the CW, CCW and OFF states of the motors. A cell updates its state by two rules, a cell makes the transition $\rightarrow+$ if it has two + neighbours, and transitions $+\rightarrow-$ and $-\rightarrow \cdot$ happen unconditionally. These simple rules generate translating localisations, or gliders.

As an initial proof-of-concept, these gliders are being used to transfer objects on the prototype surface. By using vision feedback the CA lattice is photo-excited and a glider is generated, propagating linearly. On top of this wave an object, 


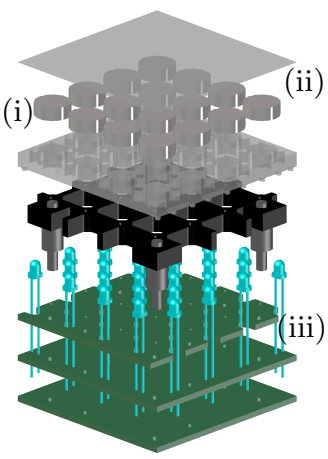

(a) Module Design

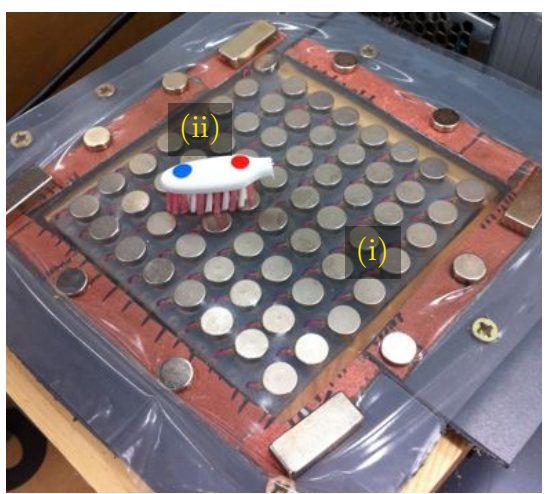

(b) Prototype

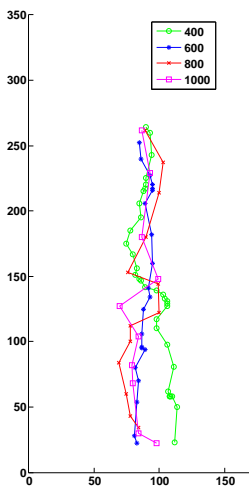

(c) Trajectories

Fig. 1. Distributed Conveyor Images

a toothbrush head particularly, Fig. 1(b)(i), is being "carried" towards a target. Toothbrush heads where used because there geometry, suitable for hygiene reasons, prove to be excellent for force manipulation, assisting in the verification process of the prototype. In Fig. 1(c) four sample trajectories (axes in pixels) are plotted with different propagation speeds (400ms, $600 \mathrm{~ms}, 800 \mathrm{~ms}, 1000 \mathrm{~ms}$ ). Repeatability of the system is sound with the variations in the trajectories be attributed by hardware manufacturing tolerances.

These initial experiments give a good indication for the performance of the proposed approach for distributed manipulation. Further work must be done to investigate more complex CA rule sets, with less specific excitation, parallel manipulation of objects and more extensive hardware modelling.

\section{References}

1. Bohringer, K.F., Donald, B.R., Mihailovich, R., MacDonald, N.C.: Theory of manipulation and control for microfabricated actuator arrays. In: IEEE Micro Electro Mechanical Systems, Oiso, Jpn, Publ by IEEE (1994) 102-107

2. Boutoustous, K., Laurent, G., Dedu, E., Matignon, L., Bourgeois, J., Le Fort-Piat, N.: Distributed control architecture for smart surfaces. In: 2010 IEEE/RSJ Int. Conf. Intelligent Robots and Systems, IROS'10, Taipei (2010) 2018-2024

3. Ataka, M., Legrand, B., Buchaillot, L., Collard, D., Fujita, H.: Design, fabrication, and operation of two-dimensional conveyance system with ciliary actuator arrays. IEEE ASME Trans Mechatron 14(1) (2009) 119-125

4. Adamatzky, A.: Computing in non-linear media and automata collectives. Institute of Physics Publishing (2001)

5. Adamatzky, A., De Lacy Costello, B., Melhuish, C., Ratcliffe, N.: Experimental reaction-diffusion chemical processors for robot path planning. J. Intel. Robot Syst 37(3) (2003) 233-249

6. Skachek, S., Adamatzky, A., Melhuish, C.: Manipulating planar shapes with a lightsensitive excitable medium: Computational studies of closed-loop systems. Int. J. Bifurcation Chaos 16(8) (2006) 2333-2349 